\title{
On Minimality of ISO Representation of Basic 2D Convolutional Codes
}

\author{
Raquel Pinto* and Rita Simões ${ }^{\bowtie}$ * \\ raquel@ua.pt ritasimoes@ua.pt \\ CIDMA - Center for Research and Development in Mathematics and Applications, \\ Department of Mathematics, University of Aveiro, \\ Campus Universitário de Santiago, 3810-193 Aveiro, Portugal
}

\begin{abstract}
In this paper we study the minimality of input-state-output (ISO) representations of basic two-dimensional (2D) convolutional codes. For that we consider the Fornasini-Marchesini ISO representations of such codes. We define the novel property of strongly modally reachable representations and we show that such representations are minimal representations of a basic $2 \mathrm{D}$ convolutional code. Moreover, we prove that the dimension of such minimal representations equals the complexity of the code.
\end{abstract}

\section{Introduction}

Two-dimensional (2D) convolutional codes are a natural generalization of onedimensional (1D) convolutional codes. These codes are naturally suitable to deal with data recorded in two dimensions, like pictures, video, data storage, etc. [9, 16, 19]. $2 \mathrm{D} / \mathrm{nD}$ convolutional codes were introduced in $[7,8,17,18]$. In $[1,3]$ the authors introduce the "locally invertible encoders" and the "Two-Dimensional Tail-Biting Convolutional Codes" with the objective of obtaining constructions of $2 \mathrm{D}$ convolutional codes with particular decoding properties. Decoding of $2 \mathrm{D}$ convolutional codes over the erasure channel was investigated in [4]. In [12] the authors define input-state-output (ISO) representations of $2 \mathrm{D}$ convolutional codes, in which the codewords of a code are generated by a $2 \mathrm{D}$ linear system.

One of the most important problems studied in the theory of convolutional codes is the minimality of representations of these codes, i.e., the determination of ISO representations with minimal dimension among all ISO representations of the code. Minimality of an ISO representation leads to more efficient practical implementations in terms of the memory space required. This problem is completely solved when we consider 1D convolutional codes $[14,15]$. However, this seems to be very hard in the $2 \mathrm{D}$ case. We address this problem by considering the Fornasini-Marchesini state-space model, originally studied in the theory of $2 \mathrm{D}$

\footnotetext{
* This work was supported by Portuguese funds through the Center for Research and Development in Mathematics and Applications (CIDMA), and The Portuguese Foundation for Science and Technology (FCT - Fundação para a Ciência e a Tecnologia), within project UID/MAT/04106/2013.
} 
linear systems [6]. Unlike the 1D case, it does not exist necessary and sufficient conditions for the minimality of a realization of a $2 \mathrm{D}$ polynomial matrix (i.e., a polynomial matrix in two indeterminates), which makes very hard to solve the general problem of minimality of ISO representations of $2 \mathrm{D}$ convolutional codes.

In this paper we consider basic 2D convolutional codes, i.e., 2D convolutional codes which are image of a zero left prime 2D polynomial matrix. We introduce the concept of strongly modally $2 \mathrm{D}$ linear system and we show that if a $2 \mathrm{D}$ basic convolutional code admits a strongly modally reachable ISO representation then this representation is minimal. Moreover, we prove that the dimension of this minimal ISO representation is equal to the complexity of the code.

\section{Convolutional Codes}

In this section we will introduce 1D and 2D convolutional codes and a representation of these codes by means of a linear system. We start by giving some preliminaries on polynomial matrices in one indeterminate and in two indeterminates that will be important for the definition of these codes.

\subsection{Polynomial matrices}

Let $\mathbb{F}$ be a field and let $\overline{\mathbb{F}}$ denote the algebraic closure of $\mathbb{F}$. Denote by $\mathbb{F}[z]$ the ring of polynomials in one indeterminate with coefficients in $\mathbb{F}$, by $\mathbb{F}(z)$ the field of fractions of $\mathbb{F}[z]$ and by $\mathbb{F}[[z]]$ the ring of formal powers series in one indeterminate with coefficients in $\mathbb{F}$.

Definition 1 (chapter 6, [10]). A matrix $U(z) \in \mathbb{F}[z]^{n \times k}$, with $n \geq k$ is,

(a) unimodular (i.e., it admits a polynomial inverse) if $n=k$ and $\operatorname{det}(U(z)) \in$ $\mathbb{F} \backslash\{0\}$;

(b) right prime $(r P)$ if for every factorization

$$
\begin{gathered}
\qquad(z)=\bar{U}(z) T(z), \\
\text { with } \bar{U}(z) \in \mathbb{F}[z]^{n \times k} \text { and } T(z) \in \mathbb{F}[z]^{n \times n}, T(z) \text { is unimodular. }
\end{gathered}
$$

A matrix is left prime $(\ell P)$ if its transpose is $r P$.

The following lemma gives characterizations of right primeness that will be needed later.

Lemma 1 (chapter 6, [10]). Let $U(z) \in \mathbb{F}[z]^{n \times k}$, with $n \geq k$. Then the following are equivalent:

(a) $U(z)$ is $r P$;

(b) there exists $P(z) \in \mathbb{F}[z]^{n \times(n-k)}$ such that $[U(z) P(z)]$ is unimodular;

(c) $U(z)$ admits a polynomial left inverse;

(d) the $k \times k$ minors of $U(z)$ have no common factor;

(e) for all $\hat{v}(z) \in \mathbb{F}(z)^{n}, \hat{v}(z)^{T} U(z) \in \mathbb{F}[z]^{n}$ implies that $\hat{v}(z) \in \mathbb{F}[z]^{n}$; 
(f) $U(\lambda)$ is full column rank, for all $\lambda \in \overline{\mathbb{F}}$.

Let us consider now polynomial matrices in two indeterminates. Denote by $\mathbb{F}\left[z_{1}, z_{2}\right]$ the ring of polynomials in two indeterminates with coefficients in $\mathbb{F}$, by $\mathbb{F}\left(z_{1}, z_{2}\right)$ the field of fractions of $\mathbb{F}\left[z_{1}, z_{2}\right]$ and by $\mathbb{F}\left[\left[z_{1}, z_{2}\right]\right]$ the ring of formal powers series in two indeterminates with coefficients in $\mathbb{F}$.

Definition 2 ([7]). A matrix $G\left(z_{1}, z_{2}\right) \in \mathbb{F}\left[z_{1}, z_{2}\right]^{n \times k}$, with $n \geq k$ is,

(a) unimodular (i.e., it admits a polynomial inverse) if $n=k$ and $\operatorname{det}\left(G\left(z_{1}, z_{2}\right)\right) \in$ $\mathbb{F} \backslash\{0\} ;$

(b) right factor prime ( $r F P$ ) if for every factorization

$$
G\left(z_{1}, z_{2}\right)=\bar{G}\left(z_{1}, z_{2}\right) T\left(z_{1}, z_{2}\right),
$$

with $\bar{G}\left(z_{1}, z_{2}\right) \in \mathbb{F}\left[z_{1}, z_{2}\right]^{n \times k}$ and $T\left(z_{1}, z_{2}\right) \in \mathbb{F}\left[z_{1}, z_{2}\right]^{k \times k}, T\left(z_{1}, z_{2}\right)$ is unimodular;

(c) right zero prime $(r Z P)$ if the ideal generated by the $k \times k$ minors of $G\left(z_{1}, z_{2}\right)$ is $\mathbb{F}\left[z_{1}, z_{2}\right]$.

A matrix is left factor prime $(\ell F P) /$ left zero prime $(\ell Z P)$ if its transpose is $r F P / r Z P$, respectively. When we consider polynomial matrices in one indeterminate, the notions (b) and (c) of the above definition are equivalent. However this is not the case for polynomial matrices in two indeterminates. In fact, zero primeness implies factor primeness, but the contrary does not happen. The following lemmas give characterizations of right factor primeness and right zero primeness that will be needed later (see $[11,13])$.

Lemma 2. Let $G\left(z_{1}, z_{2}\right) \in \mathbb{F}\left[z_{1}, z_{2}\right]^{n \times k}$, with $n \geq k$. Then the following are equivalent:

(a) $G\left(z_{1}, z_{2}\right)$ is right factor prime;

(b) for all $\hat{u}\left(z_{1}, z_{2}\right) \in \mathbb{F}\left(z_{1}, z_{2}\right)^{k}, G\left(z_{1}, z_{2}\right) \hat{u}\left(z_{1}, z_{2}\right) \in \mathbb{F}\left[z_{1}, z_{2}\right]^{n}$ implies that $\hat{u}\left(z_{1}, z_{2}\right) \in \mathbb{F}\left[z_{1}, z_{2}\right]^{k}$;

(c) the $k \times k$ minors of $G\left(z_{1}, z_{2}\right)$ have no common factor.

Lemma 3. Let $G\left(z_{1}, z_{2}\right) \in \mathbb{F}\left[z_{1}, z_{2}\right]^{n \times k}$, with $n \geq k$. Then the following are equivalent:

(a) $G\left(z_{1}, z_{2}\right)$ is right zero prime;

(b) $G\left(z_{1}, z_{2}\right)$ admits a polynomial left inverse;

(c) $G\left(\lambda_{1}, \lambda_{2}\right)$ is full column rank, for all $\lambda_{1}, \lambda_{2} \in \overline{\mathbb{F}}$.

It is well known (see [7]) that given a full column rank polynomial matrix $G\left(z_{1}, z_{2}\right) \in \mathbb{F}\left[z_{1}, z_{2}\right]^{n \times k}$, there exists a square polynomial matrix $V\left(z_{1}, z_{2}\right) \in$ $\mathbb{F}\left[z_{1}, z_{2}\right]^{k \times k}$ and a $r F P$ matrix $\bar{G}\left(z_{1}, z_{2}\right) \in \mathbb{F}\left[z_{1}, z_{2}\right]^{n \times k}$ such that

$$
G\left(z_{1}, z_{2}\right)=\bar{G}\left(z_{1}, z_{2}\right) V\left(z_{1}, z_{2}\right) .
$$




\section{CONVOLUTIONAL CODES}

The following lemma will be needed in the sequel. Let $H\left(z_{1}, z_{2}\right) \in \mathbb{F}\left[z_{1}, z_{2}\right]^{(n-k) \times n}$, $G\left(z_{1}, z_{2}\right) \in \mathbb{F}\left[z_{1}, z_{2}\right]^{n \times k}, n>k, c_{i}$ the $i$ th column of $H\left(z_{1}, z_{2}\right)$ and $r_{j}$ the $j$ th row of $G\left(z_{1}, z_{2}\right)$. We say that the full size minor of $H\left(z_{1}, z_{2}\right)$ constituted by the columns $c_{i_{1}}, \ldots, c_{i_{n-k}}$ and the full size minor of $G\left(z_{1}, z_{2}\right)$ constituted by the rows $r_{j_{1}}, \ldots, r_{j_{k}}$ are corresponding maximal order minors of $H\left(z_{1}, z_{2}\right)$ and $G\left(z_{1}, z_{2}\right)$, if

$$
\left\{i_{1}, \ldots, i_{n-k}\right\} \cup\left\{j_{1}, \ldots, j_{k}\right\}=\{1, \ldots, n\}
$$

and $\left\{i_{1}, \ldots, i_{n-k}\right\} \cap\left\{j_{1}, \ldots, j_{k}\right\}=\emptyset$.

Lemma 4 (Proposition A.4., [7]). Let $H\left(z_{1}, z_{2}\right) \in \mathbb{F}\left[z_{1}, z_{2}\right]^{(n-k) \times n}$ and $G\left(z_{1}, z_{2}\right) \in$ $\mathbb{F}\left[z_{1}, z_{2}\right]^{n \times k}$ be a $\ell F P$ and a rFP matrices, respectively, such that $H\left(z_{1}, z_{2}\right) G\left(z_{1}, z_{2}\right)=$ 0 . Then the corresponding maximal order minors of $H\left(z_{1}, z_{2}\right)$ and $G\left(z_{1}, z_{2}\right)$ are equal, modulo a unit of the ring $\mathbb{F}\left[z_{1}, z_{2}\right]$.

\subsection{D convolutional codes}

A $1 D$ (finite support) convolutional code $\mathcal{C}$ of rate $k / n$ is a (free) $\mathbb{F}[z]$-submodule of $\mathbb{F}[z]^{n}$, where $k$ is the rank of $\mathcal{C}$. A full column rank matrix $G(z) \in \mathbb{F}[z]^{n \times k}$ such that

$$
\begin{aligned}
\mathcal{C} & =\operatorname{Im}_{\mathbb{F}[z]} G(z) \\
& =\left\{\hat{v}(z) \in \mathbb{F}[z]^{n} \mid \hat{v}(z)=G(z) \hat{u}(z), \text { with } \hat{u}(z) \in \mathbb{F}[z]^{k}\right\},
\end{aligned}
$$

is called an encoder of $\mathcal{C}$. The elements of $\mathcal{C}$ are called codewords. Two full column rank matrices $G(z), \bar{G}(z) \in \mathbb{F}[z]^{n \times k}$ are equivalent encoders, i.e. they generate the same 1D convolutional code, if and only if $G(z) U(z)=\bar{G}(z)$ for some unimodular matrix $U(z) \in \mathbb{F}[z]^{k \times k}$. We denote the complexity (or degree) $\delta$ of a $1 \mathrm{D}$ convolutional code $\mathcal{C}$ as the maximum of the degree of the $k \times k$ minors of any encoder of $\mathcal{C}$ and we say that $\mathcal{C}$ is an $(n, k, \delta) 1 \mathrm{D}$ convolutional code.

Note that the fact that two equivalent encoders differ by unimodular matrices also implies that the primeness properties of the encoders of a code are preserved, i.e., if $\mathcal{C}$ admits a $r P$ encoder then all its encoders are $r P$. A $1 \mathrm{D}$ convolutional code $\mathcal{C}$ that admits a $r P$ encoder is called basic (or noncatastrophic) [14,15].

Another way of obtaining the codewords of a $1 D$ convolutional code is by means of a 1D linear system. A 1D linear system, denoted by $\Sigma=(A, B, C, D)$, is given by the updating equations

$$
\begin{aligned}
x(t+1) & =A x(t)+B u(t) \\
y(t) & =C x(t)+D u(t),
\end{aligned}
$$

where $A \in \mathbb{F}^{s \times s}, B \in \mathbb{F}^{s \times k}, C \in \mathbb{F}^{(n-k) \times s}, D \in \mathbb{F}^{(n-k) \times k}, s, n, k \in \mathbb{N}, n>k$ and with $x(0)=0$. We say that $\Sigma$ has dimension $s$. The vectors $x(t), u(t)$ and $y(t)$ represent the local state, input and output at instant $t$, respectively.

The input, state and output 1D sequences (trajectories), $\{u(t)\}_{t \in \mathbb{N}},\{x(t)\}_{t \in \mathbb{N}}$, $\{y(t)\}_{t \in \mathbb{N}}$, respectively, can be represented as formal power series:

$$
\hat{u}(z)=\sum_{t \in \mathbb{N}} u(t) z^{t} \in \mathbb{F}[[z]]^{k},
$$




$$
\begin{gathered}
\hat{x}(z)=\sum_{t \in \mathbb{N}} x(t) z^{t} \in \mathbb{F}[[z]]^{\delta}, \\
\hat{y}(z)=\sum_{t \in \mathbb{N}} y(t) z^{t} \in \mathbb{F}[[z]]^{n-k} .
\end{gathered}
$$

In the sequel we shall use the sequence and the corresponding series interchangeably.

Since the codewords of a 1D convolutional code have finite support, we will only consider the finite support input-output trajectories $(\hat{u}(z), \hat{y}(z))$ of $(1)$. Moreover, we will restrict to the finite support input-output trajectories with corresponding state trajectory $\hat{x}(z)$ also with finite support, otherwise the system would remain indefinitely excited. The finite support input-output trajectories $(\hat{u}(z), \hat{y}(z))$ with corresponding state $\hat{x}(z)$ also having finite support are called finite-weight input-output trajectories. The set of all these trajectories form a 1D convolutional code, as it is stated in the following theorem (see [15]).

Theorem 1. The set of finite-weight input-output trajectories of (1) is a $1 D$ convolutional code of rate $k / n$.

We denote by $\mathcal{C}(A, B, C, D)$ the $1 \mathrm{D}$ convolutional code whose codewords are the finite-weight input-output trajectories of the 1D linear system $\Sigma=$ $(A, B, C, D)$. Moreover, $\Sigma$ is called an input-state-output (ISO) representation of $\mathcal{C}(A, B, C, D)$. All the $1 \mathrm{D}$ convolutional codes admit (many) ISO representations. Next we will consider some properties of an ISO representation of a 1D convolutional code $\mathcal{C}$ and see how these properties are reflected on $\mathcal{C}$.

Definition 3 (chapter 6, [10]). Let $\Sigma=(A, B, C, D)$ be a $1 D$ linear system with dimension $s$.

(a) $\Sigma$ is reachable if the reachability matrix

$$
\mathcal{R}=\left[\begin{array}{lllll}
B & A B & A^{2} B & \cdots & A^{n-1} B
\end{array}\right]
$$

is full row rank, or equivalently, if the matrix $\left[\begin{array}{lll}I_{s}-A z & B z\end{array}\right]$ is $\ell P$.

(b) $\Sigma$ is observable if the observability matrix

$$
\mathcal{O}=\left[\begin{array}{c}
C \\
C A \\
\vdots \\
C A^{n-1}
\end{array}\right]
$$

is full column rank, or equivalently, if the matrix $\left[\begin{array}{c}I_{s}-A z \\ C\end{array}\right]$ is $r P$.

Theorem 2 (Lemma 2.1.1., [14]). Let $\Sigma$ be a reachable ISO representation of a $1 D$ convolutional code $\mathcal{C}$. Then $\mathcal{C}$ is basic (or noncatastrophic) if and only if $\Sigma$ is observable. 


\section{CONVOLUTIONAL CODES}

An ISO representation of a 1D convolutional code is said to be minimal if it has minimal dimension among all the ISO representations of the code. Minimality is an important property in the sense that minimal ISO representations are more efficient because they require less memory space in their implementation. Moreover, such representations have also strong structural properties which can be useful in the construction of good codes or in the implementation of decoding algorithms.

Next theorem gives a characterization of the minimal ISO representations of a 1D convolutional code and shows how these minimal ISO representations are related.

Lemma 5 (Theorem 3.4., [14]). Let $\Sigma$ be an ISO representation of an $(n, k, \delta)$ $1 D$ convolutional code $\mathcal{C}$. Then $\Sigma$ is an minimal ISO representation of $\mathcal{C}$ if and only if $\Sigma$ is reachable.

Moreover, a minimal ISO representation $\Sigma=(A, B, C, D)$ of $\mathcal{C}$ has dimension $\delta$ and any other minimal $I S O$ representation of $\mathcal{C}$ is of the form $\tilde{\Sigma}=$ $\left(S A S^{-1}, S B, C S^{-1}, D\right)$, where $S$ is a $\delta \times \delta$ invertible constant matrix.

Note that if $\Sigma$ is a minimal ISO representation of $\mathcal{C}$ then the complexity of $\mathcal{C}$ is equal to the dimension of $\Sigma$.

We can obtain a minimal ISO representation of an $(n, k, \delta) 1 \mathrm{D}$ convolutional code $\mathcal{C}$ from any ISO representation $\Sigma=(A, B, C, D)$ of $\mathcal{C}$, with dimension $s \geq \delta$. For that we consider a $s \times s$ invertible constant matrix $S$ such that

$$
S A S^{-1}=\left[\begin{array}{cc}
A_{11} & A_{12} \\
0 & A_{22}
\end{array}\right], \quad S B=\left[\begin{array}{c}
B_{1} \\
0
\end{array}\right], \quad C S^{-1}=\left[\begin{array}{ll}
C_{1} & C_{2}
\end{array}\right]
$$

where $A_{11} \in \mathbb{F}^{\delta \times \delta}, B_{1} \in \mathbb{F}^{\delta \times k}$ and $C_{1} \in \mathbb{F}^{(n-k) \times \delta}$ and $\left[I_{\delta}-A_{11} z B_{1}\right]$ is $\ell P$. Such representation is in the Kalman reachability canonical form (see [10]). Then $\Sigma_{1}=\left(A_{11}, B_{1}, C_{1}, D\right)$ is a minimal ISO representation of $\mathcal{C}$ (see [15]).

\section{$2.3 \quad 2 \mathrm{D}$ Convolutional Codes}

A $2 D$ (finite support) convolutional code $\mathcal{C}$ of rate $k / n$ is a free $\mathbb{F}\left[z_{1}, z_{2}\right]$-submodule of $\mathbb{F}\left[z_{1}, z_{2}\right]^{n}$, where $k$ is the rank of $\mathcal{C}$. A full column rank matrix $G\left(z_{1}, z_{2}\right) \in$ $\mathbb{F}\left[z_{1}, z_{2}\right]^{n \times k}$ whose columns constitute a basis for $\mathcal{C}$, i.e., such that

$$
\begin{aligned}
\mathcal{C} & =\operatorname{Im}_{\mathbb{F}\left[z_{1}, z_{2}\right]} G\left(z_{1}, z_{2}\right) \\
& =\left\{\hat{v}\left(z_{1}, z_{2}\right) \in \mathbb{F}\left[z_{1}, z_{2}\right]^{n} \mid \hat{v}\left(z_{1}, z_{2}\right)=G\left(z_{1}, z_{2}\right) \hat{u}\left(z_{1}, z_{2}\right), \text { with } \hat{u}\left(z_{1}, z_{2}\right) \in \mathbb{F}\left[z_{1}, z_{2}\right]^{k}\right\},
\end{aligned}
$$

is called an encoder of $\mathcal{C}$. The elements of $\mathcal{C}$ are called codewords. Two full column rank matrices $G\left(z_{1}, z_{2}\right), \bar{G}\left(z_{1}, z_{2}\right) \in \mathbb{F}\left[z_{1}, z_{2}\right]^{n \times k}$ are equivalent encoders if they generate the same $2 \mathrm{D}$ convolutional code, i.e., if

$$
\operatorname{Im}_{\mathbb{F}\left[z_{1}, z_{2}\right]} G\left(z_{1}, z_{2}\right)=\operatorname{Im}_{\mathbb{F}\left[z_{1}, z_{2}\right]} \bar{G}\left(z_{1}, z_{2}\right),
$$

which happens if and only if there exists a unimodular matrix $U\left(z_{1}, z_{2}\right) \in$ $\mathbb{F}\left[z_{1}, z_{2}\right]^{k \times k}$ such that $G\left(z_{1}, z_{2}\right) U\left(z_{1}, z_{2}\right)=\bar{G}\left(z_{1}, z_{2}\right)$ (see [17]). 
Note that the fact that two equivalent encoders differ by unimodular matrices also implies that the primeness properties of the encoders of a code are preserved, i.e., if $\mathcal{C}$ admits a $r F P(r Z P)$ encoder then all its encoders are $r F P(r Z P)$. A $2 \mathrm{D}$ convolutional code $\mathcal{C}$ that admits $r F P$ encoders is called noncatastrophic and it is named basic if all its encoders are $r Z P$. Finally, we denote the complexity $\delta$ of a $2 \mathrm{D}$ convolutional code $\mathcal{C}$ as the maximum of the degree of the $k \times k$ minors of any encoder of $\mathcal{C}$

2D convolutional codes can also be represented by a linear system. Unlike the $1 \mathrm{D}$ case, there are several state space models of a $2 \mathrm{D}$ linear system. In this paper we consider the Fornasini-Marchesini state-space models (see [6]). In this model a first quarter plane $2 D$ linear system, denoted by $\Sigma=\left(A_{1}, A_{2}, B_{1}, B_{2}, C, D\right)$, is given by the updating equations

$$
\begin{aligned}
x(i+1, j+1) & =A_{1} x(i, j+1)+A_{2} x(i+1, j)+B_{1} u(i, j+1)+B_{2} u(i+1, j) \\
y(i, j) & =C x(i, j)+D u(i, j),
\end{aligned}
$$

where $A_{1}, A_{2} \in \mathbb{F}^{s \times s}, B_{1}, B_{2} \in \mathbb{F}^{s \times k}, C \in \mathbb{F}^{(n-k) \times s}, D \in \mathbb{F}^{(n-k) \times k}, s, n, k \in \mathbb{N}$, $n>k$ and with past finite support of the input and of the state (i.e., $u(i, j)=0$ and $x(i, j)=0$, where 0 denotes the zero vector of appropriate lenght,t for $i<0$ or $j<0$ ) and zero initial conditions (i.e., $x(0,0)=0)$. We say that $\Sigma$ has dimension $s$. The vectors $x(i, j), u(i, j)$ and $y(i, j)$ represent the local state, input and output at $(i, j)$, respectively.

We will also represent the input, state and output 2D trajectories, $\{u(i, j)\}_{(i, j) \in \mathbb{N}^{2}}$, $\{x(i, j)\}_{(i, j) \in \mathbb{N}^{2}},\{y(i, j)\}_{(i, j) \in \mathbb{N}^{2}}$ as formal power series,

$$
\begin{gathered}
\hat{u}\left(z_{1}, z_{2}\right)=\sum_{(i, j) \in \mathbb{N}^{2}} u(i, j) z_{1}^{i} z_{2}^{j} \in \mathbb{F}\left[\left[z_{1}, z_{2}\right]\right]^{k}, \\
\hat{x}\left(z_{1}, z_{2}\right)=\sum_{(i, j) \in \mathbb{N}^{2}} x(i, j) z_{1}^{i} z_{2}^{j} \in \mathbb{F}\left[\left[z_{1}, z_{2}\right]\right]^{\delta}, \\
\hat{y}\left(z_{1}, z_{2}\right)=\sum_{(i, j) \in \mathbb{N}^{2}} y(i, j) z_{1}^{i} z_{2}^{j} \in \mathbb{F}\left[\left[z_{1}, z_{2}\right]\right]^{n-k} .
\end{gathered}
$$

For the same reasons stated for 1D convolutional codes we will restrict ourselves to finite support input-output trajectories $\left(\hat{u}\left(z_{1}, z_{2}\right), \hat{y}\left(z_{1}, z_{2}\right)\right)$ with corresponding state $\hat{x}\left(z_{1}, z_{2}\right)$ also having finite support, i.e., to the finite-weight input-output trajectories. Next theorem states that the set of these trajectories also constitute a 2D convolutional code.

Theorem 3 (Theorem 1, [12]). The set of finite-weight input-output trajectories of (2) is a $2 D$ convolutional code of rate $k / n$.

Proof. Let us denote by $S$ and $S_{i o}$ the set of finite-weight trajectories and the set of finite-weight input-output trajectories of (2), respectively. Then

$$
S=\operatorname{ker}_{\mathbb{F}\left[\left[z_{1}, z_{2}\right]\right]} X\left(z_{1}, z_{2}\right) \cap \mathbb{F}\left[z_{1}, z_{2}\right]^{n+\delta}=\operatorname{ker}_{\mathbb{F}\left(z_{1}, z_{2}\right)} X\left(z_{1}, z_{2}\right) \cap \mathbb{F}\left[z_{1}, z_{2}\right]^{n+\delta},
$$




\section{CONVOLUTIONAL CODES}

where

$$
X\left(z_{1}, z_{2}\right)=\left[\begin{array}{ccc}
I_{s}-A_{1} z_{1}-A_{2} z_{2} & -B_{1} z_{1}-B_{2} z_{2} & 0 \\
-C & -D & I_{n-k}
\end{array}\right] .
$$

Since $\operatorname{ker}_{\mathbb{F}\left(z_{1}, z_{2}\right)} X\left(z_{1}, z_{2}\right)$ has dimension $k$, there exists an $r F P$ matrix such that

$$
\operatorname{ker}_{\mathbb{F}\left(z_{1}, z_{2}\right)} X\left(z_{1}, z_{2}\right)=\operatorname{Im}_{\mathbb{F}\left(z_{1}, z_{2}\right)} \tilde{L}\left(z_{1}, z_{2}\right),
$$

and as $\tilde{L}\left(z_{1}, z_{2}\right)$ is $r F P$, we use Lemma 2 to conclude that $S=\operatorname{Im}_{\mathbb{F}\left[z_{1}, z_{2}\right]} \tilde{L}\left(z_{1}, z_{2}\right)$. Representing

$$
\tilde{L}\left(z_{1}, z_{2}\right)=\left[\begin{array}{c}
\tilde{L}_{1}\left(z_{1}, z_{2}\right) \\
\tilde{L}_{2}\left(z_{1}, z_{2}\right)
\end{array}\right]
$$

with $\tilde{L}_{1}\left(z_{1}, z_{2}\right) \in \mathbb{F}\left[z_{1}, z_{2}\right]^{\delta \times k}$ and $\tilde{L}_{2}\left(z_{1}, z_{2}\right) \in \mathbb{F}\left[z_{1}, z_{2}\right]^{n \times k}$, it follows that $S_{i o}=$ $\operatorname{Im}_{\mathbb{F}\left[z_{1}, z_{2}\right]} \tilde{L}_{2}\left(z_{1}, z_{2}\right)$. Let $F\left(z_{1}, z_{2}\right) \in \mathbb{F}\left[z_{1}, z_{2}\right]^{(\delta+n-k) \times(\delta+n-k)}$ be a nonsingular square matrix such that

$$
X\left(z_{1}, z_{2}\right)=F\left(z_{1}, z_{2}\right)\left[M_{1}\left(z_{1}, z_{2}\right) M_{2}\left(z_{1}, z_{2}\right) M_{3}\left(z_{1}, z_{2}\right)\right]
$$

where $M_{1}\left(z_{1}, z_{2}\right) \in \mathbb{F}\left[z_{1}, z_{2}\right]^{(\delta+n-k) \times \delta}, M_{2}\left(z_{1}, z_{2}\right) \in \mathbb{F}\left[z_{1}, z_{2}\right]^{(\delta+n-k) \times k}, M_{3}\left(z_{1}, z_{2}\right) \in$ $\mathbb{F}\left[z_{1}, z_{2}\right]^{(\delta+n-k) \times(n-k)}$ are such that $\left[M_{1}\left(z_{1}, z_{2}\right) M_{2}\left(z_{1}, z_{2}\right) M_{3}\left(z_{1}, z_{2}\right)\right]$ is $\ell F P$. Then

$$
\left[M_{1}\left(z_{1}, z_{2}\right) M_{2}\left(z_{1}, z_{2}\right) M_{3}\left(z_{1}, z_{2}\right)\right]\left[\begin{array}{c}
\tilde{L}_{1}\left(z_{1}, z_{2}\right) \\
\tilde{L}_{2}\left(z_{1}, z_{2}\right)
\end{array}\right]=0 .
$$

Since det $\left[\begin{array}{cc}I_{\delta}-A_{1} z_{1}-A_{2} z_{2} & 0 \\ -C & I_{n-k}\end{array}\right]$ is nonzero, it immediately follow that the we have that $\operatorname{det}\left[M_{1}\left(z_{1}, z_{2}\right) M_{3}\left(z_{1}, z_{2}\right)\right] \neq 0$ and, by Lemma 4 , the corresponding maximal order minor of $\tilde{L}_{2}\left(z_{1}, z_{2}\right)$ is also nonzero, which implies that $\tilde{L}_{2}\left(z_{1}, z_{2}\right)$ is full column rank, and therefore $S_{i o}$ is a $2 \mathrm{D}$ finite support convolutional code with rate $k / n$.

We denote by $\mathcal{C}\left(A_{1}, A_{2}, B_{1}, B_{2}, C, D\right)$ the $2 \mathrm{D}$ convolutional code whose codewords are the finite-weight input-output trajectories of the $2 \mathrm{D}$ linear system $\Sigma=\left(A_{1}, A_{2}, B_{1}, B_{2}, C, D\right)$. Moreover, $\Sigma$ is called an input-state-output (ISO) representation of $\mathcal{C}\left(A_{1}, A_{2}, B_{1}, B_{2}, C, D\right)$ (see [12]).

A 2D convolutional code admits many ISO representations and as happens in the $1 \mathrm{D}$ case, properties of the ISO representations reflect on the properties of the code. 2D linear systems as in (2) admit two types of reachability and observability notions stated in the following definition (see [6]).

Definition 4. Let $\Sigma=\left(A_{1}, A_{2}, B_{1}, B_{2}, C, D\right)$ be a $2 D$ linear system with dimension $s$.

(a) $\Sigma$ is locally reachable if the reachability matrix

$$
\mathcal{R}=\left[\begin{array}{llll}
R_{1} & R_{2} & R_{3} & \cdots
\end{array}\right] \text { is full row rank, }
$$


where $R_{k}$ represents the block matrix including all columns defined by

$$
\left(A_{1}{ }^{i-1} \uplus^{j} A_{2}\right) B_{1}+\left(A_{1}{ }^{i} \amalg^{j-1} A_{2}\right) B_{2}
$$

with $i+j=k$, for $i, j \geq 0$ and

$A_{1}{ }^{r} \uplus^{t} A_{2}=0$, when either $r$ or $t$ is negative,

$$
\begin{gathered}
A_{1}{ }^{r} \uplus^{0} A_{2}=A_{1}^{r}, \quad A_{1}{ }^{0} \uplus^{t} A_{2}=A_{2}^{t}, \text { for } r, t \geq 0, \\
A_{1}{ }^{r} \uplus^{t} A_{2}=A_{1}\left(A_{1}{ }^{r-1} \uplus^{t} A_{2}\right)+A_{2}\left(A_{1}{ }^{r} \uplus^{t-1} A_{2}\right), \text { for } r, t \geq 1 .
\end{gathered}
$$

(b) $\Sigma$ is modally reachable if the matrix

$$
\left[I_{s}-A_{1} z_{1}-A_{2} z_{2} \quad B_{1} z_{1}+B_{2} z_{2}\right]
$$

is $\ell F P$.

(c) $\Sigma$ is modally observable if the matrix

$$
\left[\begin{array}{c}
I_{s}-A_{1} z_{1}-A_{2} z_{2} \\
C
\end{array}\right]
$$

is $r F P$.

We will not consider the notion of local observability in this paper. For 1D linear systems, the notions (a) and (b) (and the corresponding observability notions) presented in the above definitions are equivalent. Such equivalence is stated in the Definition 3 (see [10]). However, this does not happen in the 2D case. There are systems which are locally reachable (observable) but not modally reachable (observable) and vice-versa (see [6]).

Given an input trajectory $\hat{u}\left(z_{1}, z_{2}\right)$ with corresponding state $\hat{x}\left(z_{1}, z_{2}\right)$ and output $\hat{y}\left(z_{1}, z_{2}\right)$ trajectories obtained from (2), the matrix

$$
\hat{r}\left(z_{1}, z_{2}\right)=\left[\begin{array}{l}
\hat{x}\left(z_{1}, z_{2}\right) \\
\hat{u}\left(z_{1}, z_{2}\right) \\
\hat{y}\left(z_{1}, z_{2}\right)
\end{array}\right]
$$

is called an input-state-output trajectory of $\Sigma=\left(A_{1}, A_{2}, B_{1}, B_{2}, C, D\right)$. The set of input-state-output trajectories of $\Sigma$ is given by

$$
\operatorname{ker}_{\mathbb{F}\left[\left[z_{1}, z_{2}\right]\right]} X\left(z_{1}, z_{2}\right)=\left\{\hat{r}\left(z_{1}, z_{2}\right) \in \mathbb{F}\left[\left[z_{1}, z_{2}\right]\right]^{s+n} \mid X\left(z_{1}, z_{2}\right) \hat{r}\left(z_{1}, z_{2}\right)=0\right\}
$$

where

$$
X\left(z_{1}, z_{2}\right)=\left[\begin{array}{ccc}
I_{s}-A_{1} z_{1}-A_{2} z_{2} & -B_{1} z_{1}-B_{2} z_{2} & 0 \\
-C & -D & I_{n-k}
\end{array}\right] \in \mathbb{F}^{(s+n-k) \times(s+n)} .
$$




\section{CONVOLUTIONAL CODES}

Moreover, there exist polynomial matrices $L\left(z_{1}, z_{2}\right) \in \mathbb{F}\left[z_{1}, z_{2}\right]^{s \times k}$ and $G\left(z_{1}, z_{2}\right) \in$ $\mathbb{F}\left[z_{1}, z_{2}\right]^{n \times k}$ such that

$$
X\left(z_{1}, z_{2}\right)\left[\begin{array}{l}
L\left(z_{1}, z_{2}\right) \\
G\left(z_{1}, z_{2}\right)
\end{array}\right]=0
$$

where $\left[\begin{array}{l}L\left(z_{1}, z_{2}\right) \\ G\left(z_{1}, z_{2}\right)\end{array}\right]$ is $r F P$ and $G\left(z_{1}, z_{2}\right)$ is an encoder of $\mathcal{C}\left(A_{1}, A_{2}, B_{1}, B_{2}, C, D\right)$ (see [12]).

The next result gives us a necessary and sufficient condition for modal reachability in terms of the matrix $X\left(z_{1}, z_{2}\right)$.

Lemma 6 ( Lemma III.4, [5]). Let $\Sigma=\left(A_{1}, A_{2}, B_{1}, B_{2}, C, D\right)$ be a $2 D$ linear system and $X\left(z_{1}, z_{2}\right)$ the corresponding matrix defined in (4). Then $\Sigma$ is modally reachable if and only if the matrix $X\left(z_{1}, z_{2}\right)$ is $\ell F P$.

If $S$ is an invertible constant matrix, it is said that the 2D linear systems

$$
\Sigma=\left(A_{1}, A_{2}, B_{1}, B_{2}, C, D\right)
$$

and

$$
\tilde{\Sigma}=\left(S A_{1} S^{-1}, S A_{2} S^{-1}, S B_{1}, S B_{2}, C S^{-1}, D\right)
$$

are algebraically equivalent (see [6]). Such systems represent the same code, as stated in the following lemma.

Lemma 7 (Proposition 4, [12]). Let $\Sigma=\left(A_{1}, A_{2}, B_{1}, B_{2}, C, D\right)$ be a $2 D$ linear system with dimension $s$ and $S$ a $s \times s$ invertible constant matrix. Then

$$
\begin{gathered}
\mathcal{C}\left(A_{1}, A_{2}, B_{1}, B_{2}, C, D\right)= \\
=\mathcal{C}\left(S A_{1} S^{-1}, S A_{2} S^{-1}, S B_{1}, S B_{2}, C S^{-1}, D\right) .
\end{gathered}
$$

An ISO representation of a $2 \mathrm{D}$ convolutional code is said to be minimal if it has minimal dimension among all the ISO representations of the code. Also in [6], Fornasini and Marchesini generalized the Kalman reachability canonical form for 2D linear systems, considered in the next definition, and showed that every $2 \mathrm{D}$ linear system is algebraically equivalent to a system in the Kalman reachability form.

Definition 5 ([6]). A $2 D$ linear system $\Sigma=\left(A_{1}, A_{2}, B_{1}, B_{2}, C, D\right)$, with dimension $s, k$ inputs and $n-k$ outputs is in the Kalman reachability canonical form if

$A_{1}=\left[\begin{array}{cc}A_{11}^{(1)} & A_{12}^{(1)} \\ 0 & A_{22}^{(1)}\end{array}\right], \quad A_{2}=\left[\begin{array}{cc}A_{11}^{(2)} & A_{12}^{(2)} \\ 0 & A_{22}^{(2)}\end{array}\right], \quad B_{1}=\left[\begin{array}{c}B_{1}^{(1)} \\ 0\end{array}\right], \quad B_{2}=\left[\begin{array}{c}B_{1}^{(2)} \\ 0\end{array}\right], \quad C=\left[\begin{array}{ll}C_{1} & C_{2}\end{array}\right]$

where $A_{11}^{(1)}, A_{11}^{(2)} \in \mathbb{F}^{\delta \times \delta}, B_{1}^{(1)}, B_{1}^{(2)} \in \mathbb{F}^{\delta \times k}, C_{1} \in \mathbb{F}^{(n-k) \times \delta}$, with $s \geq \delta$ and the remaining matrices of suitable dimensions, and $\Sigma_{1}=\left(A_{11}^{(1)}, A_{11}^{(2)}, B_{1}^{(1)}, B_{1}^{(2)}, C_{1}, D\right)$ is a locally reachable system, which is the largest locally reachable subsystem of $\Sigma$. 
Proposition 1 (Proposition 4, [12]). Let $\Sigma=\left(A_{1}, A_{2}, B_{1}, B_{2}, C, D\right)$ be a ISO representation of a $2 D$ convolutional code $\mathcal{C}$. Let $S$ be an invertible constant matrix such that

$$
\tilde{\Sigma}=\left(S A_{1} S^{-1}, S A_{2} S^{-1}, S B_{1}, S B_{2}, C S^{-1}, D\right)
$$

is in the Kalman reachability canonical form and let

$$
\tilde{\Sigma}_{1}=\left(\tilde{A}_{11}^{(1)}, \tilde{A}_{11}^{(2)}, \tilde{B}_{1}^{(1)}, \tilde{B}_{1}^{(2)}, \tilde{C}_{1}, D\right)
$$

be the largest locally reachable subsystem of $\tilde{\Sigma}$. Then $\mathcal{C}=\mathcal{C}\left(\tilde{A}_{11}^{(1)}, \tilde{A}_{11}^{(2)}, \tilde{B}_{1}^{(1)}, \tilde{B}_{1}^{(2)}, \tilde{C}_{1}, D\right)$.

The next result follows immediately.

Corollary 1. Minimal ISO representations of a $2 D$ convolutional code must be locally reachable.

However, it does not exist a sufficient condition for minimality of ISO representations of a 2D convolutional code. In fact, minimality of these ISO representations is a hard problem investigated by many authors which has been open for many decades. In the next section we will investigate minimality of ISO representations of basic $2 \mathrm{D}$ convolutional codes and we will obtain a sufficient condition for an ISO representation to be minimal.

\section{On Minimality of ISO Representations of Basic 2D Convolutional Codes}

In this section we only consider basic $2 \mathrm{D}$ convolutional codes.

Let $\Sigma=\left(A_{1}, A_{2}, B_{1}, B_{2}, C, D\right)$ be a modally reachable ISO representation of a basic $2 \mathrm{D}$ convolutional code $\mathcal{C}$ and let $G\left(z_{1}, z_{2}\right)$ be an encoder of $\mathcal{C}$. Then

$$
X\left(z_{1}, z_{2}\right)\left[\begin{array}{l}
L\left(z_{1}, z_{2}\right) \\
G\left(z_{1}, z_{2}\right)
\end{array}\right]=0
$$

where $X\left(z_{1}, z_{2}\right)$ is defined in (4) and $L\left(z_{1}, z_{2}\right)$ is a suitable polynomial matrix. Since $G\left(z_{1}, z_{2}\right)$ is $r Z P$, so it is $\left[\begin{array}{l}L\left(z_{1}, z_{2}\right) \\ G\left(z_{1}, z_{2}\right)\end{array}\right]$ and, by Definition 2 and Lemma 4 , $X\left(z_{1}, z_{2}\right)$ must be $\ell Z P$.

Next lemma relates the property of left zero primeness of the matrix $X\left(z_{1}, z_{2}\right)$ of a 2 D linear system $\Sigma$ with a special type of modal reachability of $\Sigma$.

Lemma 8. Let $A_{1}, A_{2} \in \mathbb{F}^{s \times s}, B_{1}, B_{2} \in \mathbb{F}^{s \times k}, C \in \mathbb{F}^{(n-k) \times s}, D \in \mathbb{F}^{(n-k) \times k}$, $s, n, k \in \mathbb{N}, n>k$. Then

$$
\left[I_{s}-A_{1} z_{1}-A_{2} z_{2} \quad B_{1} z_{1}+B_{2} z_{2}\right]
$$

is $\ell Z P$ if and only if the corresponding matrix $X\left(z_{1}, z_{2}\right)$ defined in (4) is $\ell Z P$. 
Proof. Suppose that the matrix $\left[I_{s}-A_{1} z_{1}-A_{2} z_{2} \quad B_{1} z_{1}+B_{2} z_{2}\right]$ is $\ell Z P$; then there exist $U_{1}\left(z_{1}, z_{2}\right) \in \mathbb{F}\left[z_{1}, z_{2}\right]^{s \times s}$ and $U_{2}\left(z_{1}, z_{2}\right) \in \mathbb{F}\left[z_{1}, z_{2}\right]^{k \times s}$ such that

$$
\left[\begin{array}{ll}
I_{s}-A_{1} z_{1}-A_{2} z_{2} & B_{1} z_{1}+B_{2} z_{2}
\end{array}\right]\left[\begin{array}{l}
U_{1}\left(z_{1}, z_{2}\right) \\
U_{2}\left(z_{1}, z_{2}\right)
\end{array}\right]=I_{s}
$$

So

$$
\left[\begin{array}{ccc}
I_{s}-A_{1} z_{1}-A_{2} z_{2} & -B_{1} z_{1}-B_{2} z_{2} & 0 \\
-C & -D & I_{n-k}
\end{array}\right]\left[\begin{array}{cc}
U_{1}\left(z_{1}, z_{2}\right) & 0 \\
-U_{2}\left(z_{1}, z_{2}\right) & 0 \\
C U_{1}\left(z_{1}, z_{2}\right)-D U_{2}\left(z_{1}, z_{2}\right) & I_{n-k}
\end{array}\right]=I_{s+n-k}
$$

and therefore $X\left(z_{1}, z_{2}\right)$ is $\ell Z P$. The other implication follows trivially.

This means that ISO representations $\Sigma=\left(A_{1}, A_{2}, B_{1}, B_{2}, C, D\right)$ with dimension $s$ of $2 \mathrm{D}$ basic convolutional codes which are modally reachable are such that $\left[I_{s}-A_{1} z_{1}-A_{2} z_{2} B_{1} z_{1}+B_{2} z_{2}\right]$ is $\ell Z P$. This property will very important throughout the paper. So we propose the next definition.

Definition 6. Let $\Sigma=\left(A_{1}, A_{2}, B_{1}, B_{2}, C, D\right)$ be a $2 D$ linear system with dimension s. $\Sigma$ is said to be strongly modally reachable if $\left[I_{s}-A_{1} z_{1}-A_{2} z_{2} \quad B_{1} z_{1}+B_{2} z_{2}\right]$ is $\ell Z P$.

It is obvious that strongly modally reachable systems are also modally reachable, but the converse is not true.

Next we will consider the projections of a $2 \mathrm{D}$ convolutional code $\mathcal{C}$ onto the two semi-axis $\left\{\ell e_{i} \mid \ell \in \mathbb{N}\right\}$, for $i=1,2$, with $e_{1}=(1,0)$ and $e_{2}=(0,1)$, respectively:

$$
\mathcal{C}_{1}=\operatorname{proj}_{z_{1}} \mathcal{C}=\left\{\hat{v}\left(z_{1}, 0\right): \hat{v}\left(z_{1}, z_{2}\right) \in \mathcal{C}\right\}
$$

and

$$
\mathcal{C}_{2}=\operatorname{proj}_{z_{2}} \mathcal{C}=\left\{\hat{v}\left(0, z_{2}\right): \hat{v}\left(z_{1}, z_{2}\right) \in \mathcal{C}\right\} .
$$

$\mathcal{C}_{1}$ and $\mathcal{C}_{2}$ are $1 \mathrm{D}$ convolutional codes (see [15]). Moreover, if $G\left(z_{1}, z_{2}\right) \in \mathbb{F}\left[z_{1}, z_{2}\right]^{n \times k}$ is an encoder of $\mathcal{C}$ then

$$
\mathcal{C}_{1}=\operatorname{Im}_{\mathbb{F}\left[z_{1}\right]} G\left(z_{1}, 0\right) \quad \text { and } \quad \mathcal{C}_{2}=\operatorname{Im}_{\mathbb{F}\left[z_{2}\right]} G\left(0, z_{2}\right)
$$

Note that $G\left(z_{1}, 0\right)$ or $G\left(0, z_{2}\right)$ may not have full column rank and therefore they may not be encoders of $\mathcal{C}_{1}$ and $\mathcal{C}_{2}$, respectively. Furthermore, the noncatastrophicity of $\mathcal{C}$ does not imply the noncatastrophicity of $\mathcal{C}_{1}$ and $\mathcal{C}_{2}$ (see [12]). However, if $\mathcal{C}$ is basic then $\mathcal{C}_{1}$ and $\mathcal{C}_{2}$ are basic. In fact, if $G\left(z_{1}, z_{2}\right)$ is $r Z P$ then there exists $Y\left(z_{1}, z_{2}\right) \in \mathbb{F}\left[z_{1}, z_{2}\right]^{k \times n}$ such that

$$
Y\left(z_{1}, z_{2}\right) G\left(z_{1}, z_{2}\right)=I_{k} \Rightarrow Y\left(z_{1}, 0\right) G\left(z_{1}, 0\right)=I_{k}
$$

i.e., $G\left(z_{1}, 0\right)$ is $r P$ and then $\mathcal{C}_{1}$ is basic and $G\left(z_{1}, 0\right)$ is its encoder. Analogously, we prove that $\mathcal{C}_{2}$ is basic and $G\left(0, z_{2}\right)$ is its encoder. Moreover, if $\mathcal{C}$ has rate $k / n$, $\mathcal{C}_{1}$ and $\mathcal{C}_{2}$ also have rate $k / n$. 
Furthermore, let $\Sigma=\left(A_{1}, A_{2}, B_{1}, B_{2}, C, D\right)$ be an ISO representation of a $2 \mathrm{D}$ convolutional code $\mathcal{C}$ and consider the restriction of a trajectory of $\{x(i, j), u(i, j), y(i, j)\}(i, j) \in \mathbb{N}^{2}$ of $\Sigma$ to the semi-axis $\left\{\ell e_{1} \mid \ell \in \mathbb{N}\right\}$, i.e., $\{x(i, 0), u(i, 0), y(i, 0)\}_{i \in \mathbb{N}}$. By the zero initial condition and the past finite support property of the input and state, we have that

$$
\begin{aligned}
x(i+1,0) & =A_{1} x(i, 0)+B_{1} u(i, 0) \\
y(i, 0) & =C x(i, 0)+D u(i, 0)
\end{aligned}
$$

with $x(0,0)=0$. This means that the $1 \mathrm{D}$ linear system $\Sigma_{1}=\left(A_{1}, B_{1}, C, D\right)$ generates the restrictions to the semi-axes $\left\{\ell e_{1} \mid \ell \in \mathbb{N}\right\}$, of all trajectories of $\Sigma$, i.e., $\Sigma_{1}$ is an ISO representation of $\mathcal{C}_{1}$, and analogously, $\Sigma_{2}=\left(A_{2}, B_{2}, C, D\right)$ is an ISO representation of $\mathcal{C}_{2}$.

Theorem 4. Let $\Sigma=\left(A_{1}, A_{2}, B_{1}, B_{2}, C, D\right)$ be a strongly modally reachable ISO representation of a $2 D$ convolutional code with dimension $s$. Then $\Sigma$ is a minimal ISO representation.

Proof. By the previous lemma, $X\left(z_{1}, z_{2}\right)$ is $\ell Z P$ and therefore $X\left(z_{1}, 0\right)$ and $X\left(0, z_{2}\right)$ are $\ell P$. In fact, if $X\left(z_{1}, z_{2}\right)$ is $\ell Z P$ then there exists $\tilde{X}\left(z_{1}, z_{2}\right) \in$ $\mathbb{F}\left[z_{1}, z_{2}\right]^{(s+n) \times(s+n-k)}$ such that $X\left(z_{1}, z_{2}\right) \widetilde{X}\left(z_{1}, z_{2}\right)=I_{s+n-k}$ which implies that

$$
X\left(z_{1}, 0\right) \tilde{X}\left(z_{1}, 0\right)=I_{s+n-k} \quad \text { and } \quad X\left(0, z_{2}\right) \tilde{X}\left(0, z_{2}\right)=I_{s+n-k}
$$

which means that

$X\left(z_{1}, 0\right)=\left[\begin{array}{ccc}I_{s}-A_{1} z_{1} & -B_{1} z_{1} & 0 \\ -C & -D & I_{n-k}\end{array}\right]$ and $X\left(0, z_{2}\right)=\left[\begin{array}{ccc}I_{s}-A_{2} z_{2} & -B_{2} z_{2} & 0 \\ -C & -D & I_{n-k}\end{array}\right]$

are $\ell P$.

Then $\left[I_{s}-A_{1} z \quad B_{1} z\right]$ and $\left[I_{s}-A_{2} z \quad B_{2} z\right]$ are $\ell P$ and therefore, by Definition $3, \Sigma_{1}=\left(A_{1}, B_{1}, C, D\right)$ and $\Sigma_{2}=\left(A_{2}, B_{2}, C, D\right)$ are reachable. Thus, by Lemma $5, \Sigma_{i}$ is a minimal ISO representation of $\mathcal{C}_{i}=\mathcal{C}\left(A_{i}, B_{i}, C, D\right)$, for $i=1,2$.

Now suppose that $\Sigma$ is not a minimal ISO representation of $\mathcal{C}$. Then there exists $\widetilde{\Sigma}=\left(\widetilde{A}_{1}, \widetilde{A}_{2}, \widetilde{B}_{1}, \widetilde{B}_{2}, \widetilde{C}, \widetilde{D}\right)$ a minimal ISO representation of $\mathcal{C}$ with dimension $\widetilde{s}<s$. Then, for $i=1,2, \widetilde{\Sigma}_{i}=\left(\widetilde{A}_{i}, \widetilde{B}_{i}, \widetilde{C}, \widetilde{D}\right)$ is an ISO representation of $\mathcal{C}_{i}$ with smaller dimension than $\Sigma_{i}$, which contradicts the fact that $\Sigma_{i}$ is a minimal ISO representation of $\mathcal{C}_{i}$. Then $\Sigma$ is a minimal ISO representation of $\mathcal{C}$.

The next results follow immediately. It shows that if $\mathcal{C}$ is a basic $2 \mathrm{D}$ convolutional code with a strongly modally reachable ISO representation $\Sigma=$ $\left(A_{1}, A_{2}, B_{1}, B_{2}, C, D\right)$, then the complexity of $\mathcal{C}$ is equal to the dimension of a minimal ISO representation of $\mathcal{C}$.

Corollary 2. Let $\mathcal{C}$ be a basic $2 D$ convolutional code of rate $k / n$ with a strongly modally reachable $I S O$ representation $\Sigma$ of dimension $s$. Then $\mathcal{C}$ has complexity $s$. Moreover, the projections of $\mathcal{C}$ onto the semi-axes $\left\{\ell e_{1} \mid \ell \in \mathbb{N}\right\}$ and $\left\{\ell e_{2} \mid \ell \in \mathbb{N}\right\}$, respectively have rate $k / n$ and complexity $s$. 
Proof. Let us assume that $\Sigma=\left(A_{1}, A_{2}, B_{1}, B_{2}, C, D\right)$ is a strongly modally reachable ISO representation of $\mathcal{C}$ with dimension $s$. By the above theorem $\Sigma$ is a minimal ISO representation of $\mathcal{C}$ and $\Sigma_{1}=\left(A_{1}, B_{1}, C, D\right)$ and $\Sigma_{2}=$ $\left(A_{2}, B_{2}, C, D\right)$ are also minimal ISO representations of $\mathcal{C}_{1}$ and $\mathcal{C}_{2}$, respectively, with dimension $\delta$. Then, by Lemma $5, \mathcal{C}_{1}$ and $\mathcal{C}_{2}$ have complexity $s$.

Let $G\left(z_{1}, z_{2}\right)$ be an encoder of $\mathcal{C}$ and $L\left(z_{1}, z_{2}\right)$ a suitable polynomial matrix such that

$$
X\left(z_{1}, z_{2}\right)\left[\begin{array}{l}
L\left(z_{1}, z_{2}\right) \\
G\left(z_{1}, z_{2}\right)
\end{array}\right]=0
$$

where $X\left(z_{1}, z_{2}\right)$ is defined in (4). Since the full size minors of $X\left(z_{1}, z_{2}\right)$ have degree smaller or equal than $s$, it follows from Lemma 4 that also the full size minors of $G\left(z_{1}, z_{2}\right)$ have degree less or equal than $s$. On the other hand, as $\mathcal{C}_{1}$ has complexity $s, G\left(z_{1}, 0\right)$ is an encoder of $\mathcal{C}_{1}$ that has one full size minor of degree $s$ and therefore $G\left(z_{1}, z_{2}\right)$ has one full size minor of degree greater or equal than $s$. Conseguently, the greatest degree of the full size minors of $G\left(z_{1}, z_{2}\right)$ is $s$ and therefore $\mathcal{C}$ has complexity $s$.

Corollary 3. Let $\Sigma$ be a strongly modally reachable $2 D$ linear system. Then $\Sigma$ is locally reachable.

Proof. It follows from Corollary 1.

\section{Conclusion}

In this paper we have investigated the minimality of ISO representations of basic $2 \mathrm{D}$ convolutional codes. We have showed that if a basic $2 \mathrm{D}$ convolutional code admits a strongly modally reachable ISO representation then this ISO representation is minimal with dimension equal to the complexity of the code. This result is a natural generalization of the characterization of minimal ISO representations of basic (or noncatastrophic) 1D convolutional codes. We believe that all basic 2D convolutional codes admit a strongly modally reachable ISO representation and that, as happens in the $1 \mathrm{D}$ case, all minimal ISO representations of a basic $2 \mathrm{D}$ convolutional code are algebraically equivalent. We will investigate this problem in the future. For that we will make use of the so-called first order representations of a code.

\section{References}

1. L. Alfandary and D. Raphaeli, "Ball codes - Two-dimensional tail-biting convolutional codes", Proceedings 2010 IEEE Global Communications Conference (GLOBECOM 2010), Miami, FL, 2010.

2. S. Benedetto, D. Divsalar, G. Montorsi and F. Pollara, "Serial concatenation of interleaved codes: Performance analysis, design, and iterative decoding", IEEE Transactions on Information Theory vol. 44, no. 3, pp. 909-926, 1998. 
3. C. Charoenlarpnopparut, "Applications of Gr'obner bases to the structural description and realization of multidimensional convolutional code", ScienceAsia, 35, pp. 95-105, 2009.

4. J.-J. Climent, D. Napp, R. Pinto and R. Simões, "Decoding of 2D convolutional codes over the erasure channel", Advances in Mathematics of Communications, vol. 10(1), pp. 179-193, 2016.

5. J.-J. Climent, D. Napp, R. Pinto and R. Simões, "Series concatenation of 2D convolutional codes", Proceedings IEEE 9th International Workshop on Multidimensional $(n D)$ Systems (nDS), Vila Real, Portugal, 2015.

6. E. Fornasini and G. Marchesini, "Structure and properties of two-dimensional systems", Multidimensional Systems, Techniques and Applications, editor: S. G. Tzafestas, Electrical and Computer Engineering, vol. 29, pp. 37-88, 1986.

7. E. Fornasini and M. E. Valcher, "Algebraic aspects of two-dimensional convolutional codes", IEEE Transactions on Information Theory, vol. 40, no. 4, pp. 1068 1082, 1994.

8. H. Gluesing-Luersen, J. Rosenthal and P. A. Weiner, "Duality between mutidimensinal convolutional codes and systems", Advances in Mathematical Systems Theory, A Volume in Honor of Diedrich Hinrichsen, editors: F. Colonius, U. Helmke, F. Wirth, and D. Pr'atzel-Wolters, pp. 135-150, Birkh'auser, Boston, 2000 .

9. J. Justesen and S. Forchhammer, "Two dimensional information theory and coding: with applications to graphics data and high-density storage media", Cambridge University Press, Cambridge, UK, 2010.

10. T. Kailath, Linear Systems. Prentice-Hall, Englewood Cliffs, NJ, 1980.

11. B.C. Lévy, "2d-polynomial and rational matrices and their applications for the modelling of 2-d dynamical systems", Ph.D. dissertation, Department of Electrical Engineering, Stanford University, Stanford, CA, 1981.

12. D. Napp, C. Perea and R. Pinto, "Input-state-output representations and constructions of finite support 2D convolutional codes", Advances in Mathematics of Communications, vol. 4, no. 4, pp. 533-545, 2010.

13. P. Rocha, "Structure and representation of 2-d systems", Ph.D. dissertation, University of Groningen, Groningen, The Netherlands, 1990.

14. J. Rosenthal, J.M. Schumacher and E.V. York, "On behaviors and convolutional codes", IEEE Transactions on Information Theory, vol. 42, no. 6, pp. 1881-1891, 1996.

15. J. Rosenthal and E.V. York, "BCH convolutional codes", IEEE Transactions on Information Theory, vol. 45, no. 6, pp. 1833-1844, 1999.

16. J. Singh and M. L. Singh, "A new family of two-dimensional codes for optical CDMA systems", Optik - Interntional Journal Light and Electron Optics, vol. 120 (18), pp. 959-962, 2009.

17. M.E. Valcher and E. Fornasini, "On 2D finite support convolutional codes: An algebraic approach", Multidimensional Systems and Signal Processing, vol. 5, pp. 231-243, 1994.

18. P. A. Weiner, "Multidimensional convolutional codes", Ph.D. dissertation, Department of Mathematics, University of Notre Dame, Indiana, USA, 1998.

19. X.-L. Zhou and Y. Hu, "Multilength two-dimensional codes for optical CDMA systems", Optoelectronics Letters, vol. 1 (3), pp 232-234, 2005. 